\title{
Cannabinoid-1 receptor deletion in podocytes mitigates both glomerular and tubular dysfunction in a mouse model of diabetic nephropathy
}

\author{
Tony Jourdan ${ }^{1 \#^{*}}$, Joshua K. Park ${ }^{1}$, Zoltán V. Varga² ${ }^{2}$ János Pálóczi $^{2}$, Nathan J. \\ Coffey ${ }^{1}$, Avi Z. Rosenberg ${ }^{3,4}$, Grzegorz Godlewski ${ }^{1}$, Resat Cinar ${ }^{1}$, Ken Mackie $^{5}$, \\ Pal Pacher ${ }^{2}$ and George Kunos ${ }^{1 *}$
}

${ }^{1}$ Laboratory of Physiologic Studies, National Institute on Alcohol Abuse and Alcoholism (NIAAA), National Institutes of Health (NIH), Bethesda, MD 20892; ${ }^{2}$ Laboratory of Cardiovascular Physiology and Tissue Injury, NIH/NIAAA, Bethesda, MD, 20852, USA.

${ }^{3}$ Department of Pathology Johns Hopkins University, Baltimore MD and ${ }^{4}$ Kidney Diseases Section, National Institute on Diabetes, Digestive, and Kidney Diseases (NIDDK), NIH, Bethesda, MD 20892. ${ }^{5}$ Department of Psychological and Brain Sciences, Indiana University, Bloomington, IN 47405.

\# After December 1st 2017, Team Pathophysiology of Dyslipidemia, INSERM UMR1231

Lipids, Nutrition, Cancer, Université de Bourgogne Franche-Comté, Dijon, France. @: tony.jourdan@inserm.fr

*Correspondence: Tony Jourdan, Ph.D. or George Kunos, M.D., Ph.D.

National Institute on Alcohol Abuse and Alcoholism, NIH, 5625 Fishers Lane, MSC-9413, Bethesda, MD 20892-9413, USA. Tel: 301-443-2069, Fax: 301-480-0257. E-mail:

tony.jourdan@nih.gov or george.kunos@nih.gov

Keywords: endocannabinoid, podocyte, hyperglycemia, tubular function

\section{Abstract}

\section{Aims}

To determine the specific role of podocyte-expressed cannabinoid-1 receptor $\left(\mathrm{CB}_{1} \mathrm{R}\right)$ in the development of diabetic nephropathy $(\mathrm{DN})$, relative to $\mathrm{CB}_{1} \mathrm{R}$ in other renal cell types.

\section{Material and methods}

This article has been accepted for publication and undergone full peer review but has not been through the copyediting, typesetting, pagination and proofreading process, which may lead to differences between this version and the Version of Record. Please cite this article as doi: 10.1111/dom.13150 
We developed a mouse model with a podocyte-specific deletion of $\mathrm{CB}_{1} \mathrm{R}$ (pCB1Rko) and challenged this model with streptozotocin (STZ)-induced type-1 DN. We also assessed the podocyte response to high glucose in vitro and its effects on $\mathrm{CB}_{1} \mathrm{R}$ activation.

\section{Results}

High glucose exposure for $48 \mathrm{~h}$ led to an increase in $\mathrm{CB}_{1} \mathrm{R}$ gene expression $(C N R 1)$ and endocannabinoid production in cultured human podocytes. This was associated with podocyte injury reflected by decreased podocin and nephrin expression. These changes could be prevented by Cnr1-silencing, thus identifying CB1R as a key player in podocyte injury. After 12 weeks of chronic hyperglycemia, STZ-treated pCB1Rko mice showed similar elevated blood glucose as their wild-type littermates. However, they displayed less albuminuria and less podocyte loss than STZ-treated wild-type mice. Unexpectedly, pCB1Rko mice also have milder tubular dysfunction, fibrosis and reduction of cortical microcirculation compared to wild-type controls, which is partly mediated by podocyte-derived endocannabinoids acting via $\mathrm{CB}_{1} \mathrm{R}$ on proximal tubular cells.

\section{Conclusions}

Activation of $\mathrm{CB}_{1} \mathrm{R}$ in podocytes contributes to both glomerular and tubular dysfunction in type$1 \mathrm{DN}$, which highlights the therapeutic potential of peripheral $\mathrm{CB}_{1} \mathrm{R}$ blockade. 


\section{Introduction}

Diabetes mellitus (hereafter referred to as diabetes) has reached pandemic proportions with an estimated 415 million individuals suffering from diabetes worldwide in 2015 , which is projected to increase to 642 million by 2040. DN, is a leading cause of chronic kidney disease (CKD) and end-stage renal disease and contributes to excess morbidity and mortality. DN is a microvascular complication of both type-1 and type-2 diabetes (T1D, T2D) and characterized by dysfunction and injury to the glomerular filtration barrier, leading to albuminuria. Chronic injury leads to glomerulosclerosis, interstitial fibrosis and tubular atrophy, which manifests clinically as a reduction in glomerular filtration rate $[1,2]$.

Podocytes are highly specialized epithelial cells that wrap around the glomerular tuft in juxtaposition to the glomerular basement membranes thereby covering the glomerular capillaries. Podocyte foot processes form slits that represent one layer of the glomerular filtration barrier and enable the efficient ultrafiltration of blood to form tubular fluid [3]. The structural integrity of podocytes is key for proper glomerular function and podocyte injury underlies the progression of several renal pathologies [4]. Additionally, podocytes interact with mesangial and glomerular endothelial cells through various paracrine mediators to maintain normal glomerular functions and disruption in this cellular cross-talk may contribute to $\mathrm{DN}[5,6]$.

The endocannabinoid system (ECS) is mainly comprised of 2 membrane receptors $\left(\mathrm{CB}_{1} \mathrm{R}\right.$ and $\mathrm{CB}_{2} \mathrm{R}$ ) and their 2 major endogenous ligands, $\mathrm{N}$-arachidonoyl-ethanolamine (anandamide or AEA) and 2-arachidonoyl-glycerol (2-AG). In addition to its well-documented role in obesity and its metabolic complications [7], the ECS has been implicated in the pathogenesis of CKD including $\mathrm{DN}[8,9]$. Both $\mathrm{CB}_{1} \mathrm{R}$ and $\mathrm{CB}_{2} \mathrm{R}$ are expressed in the human and rodent kidney [8], particularly in mesangial cells [10], podocytes [11], and proximal tubular cells [12, 13]. Enzymes responsible for 
the biosynthesis and degradation of endocannabinoids are also expressed in the kidney $[9,14]$. We have previously reported that $\mathrm{CB}_{1} \mathrm{R}$ activation in podocytes mediates podocyte injury caused by hyperglycemia and increased RAAS activity in a rat model of T2D [11]. Furthermore, in vitro exposure of podocytes to high glucose increases $\mathrm{CB}_{1} \mathrm{R}$ expression, and this effect is abrogated by $\mathrm{CB}_{1} \mathrm{R}$ blockade [15]. However, the specific role of $\mathrm{CB}_{1} \mathrm{R}$ in podocytes in the development of $\mathrm{DN}$, relative to $\mathrm{CB}_{1} \mathrm{R}$ in other renal cell types remains unclear. Here we report that podocyte-specific genetic deletion of $\mathrm{CB}_{1} \mathrm{R}$ partially protects against the streptozotocin-induced glomerular dysfunction and, surprisingly, also prevents proximal tubular injury, possibly by improving the renal microcirculation and also by preventing the release of humoral factors involved in tubular damage from podocytes. These observations highlight the key pathogenic role of podocyte $\mathrm{CB}_{1} \mathrm{R}$ in DN.

\section{RESULTS}

\section{High-glucose activates the ECS in podocytes and promotes podocyte injury.}

Chronic exposure of human cultured podocytes to high-glucose $(30 \mathrm{mM})$ elicited a significant increase in CNR1 but not CNR2 expression within $48 \mathrm{~h}$, as compared to cells exposed to $5 \mathrm{mM}$ glucose. This was accompanied by an increase in the cellular levels of AEA and 2-AG (Figure 1A). High-glucose exposure also decreased gene expression of podocin (NPHS2) and nephrin (NPHS1), proteins involved in maintaining podocyte integrity, while increasing gene expression of desmin (DES), a protein marker of podocyte injury, after $48 \mathrm{~h}$ and $72 \mathrm{~h}$ (Figure 1B). Furthermore, $48 \mathrm{~h}$ exposure to $30 \mathrm{mM}$ glucose increased podocyte-secretion of pro-inflammatory cytokines, including Chemokine Ligand-2 (CCL2, also called monocyte chemoattractant protein-1; MCP-1), tumor necrosis factor- $\alpha$ (TNF- $\alpha$ ), and interleukin-18 (IL-18) (Figure 1C). Furthermore, gene expression of transforming growth factor $\beta 1$ (TGFB1) was similarly strongly increased (Figure 
1D). To determine if these changes were related to $\mathrm{CB}_{1} \mathrm{R}$ activation, we exposed podocytes to 30 $\mathrm{mM}$ high-glucose for $48 \mathrm{~h}$ in the presence of CNR1-siRNA. Treatment with CNR1-siRNA completely abolished the high-glucose-induced CNR1 overexpression, reducing CNR1 mRNA significantly below that of cells maintained in normal glucose, without influencing CNR2 expression (Figure 1E). In addition, CNR1 knock-down completely prevented the high-glucoseinduced podocyte injury, as indicated by the normalization of podocin (NPHS2), nephrin (NPHS1) and desmin (DES) gene expression (Figure 1F). The high-glucose-mediated secretion of CCL2, TNF- $\alpha$, IL-18, and TGFB1 expression were also significantly attenuated by CNR1 knockdown (Figure 1E, 1G). To establish whether these observations were relevant in vivo, we developed a mouse model with a podocyte-specific deletion of Cnr1.

\section{Podocyte-specific deletion of $\mathrm{Cnr} 1$ mitigates the loss of glomerular function in streptozotocin- treated diabetic mice.}

Deletion of Cnr1 in podocytes was first assessed by cell-specific genotyping. Podocytes-nuclei stained for the marker Wilms' tumor-1 (WT-1) were laser captured using the xMD method. Compared to wild-type podocytes, pCB1Rko podocytes showed a shift in the DNA band for Cnr1 in WT-1 ${ }^{+}$but not WT-1 ${ }^{-}$nuclei (Supplementary figure 1A), indicating podocyte-specific deletion of Cnr1. The absence of $\mathrm{CB}_{1} \mathrm{R}$ protein in podocytes was confirmed by co-localization with podocalyxin as visualized by double immuno-fluorescence/confocal microscopy (Supplementary figure 1B). The phenotype of pCB1Rko mice was indistinguishable from that of their wild-type $\mathrm{Cre}^{+}$littermates, displaying comparable body and kidney weights and normal parameters of kidney function. They also had similar endocannabinoids levels, but half as much $\mathrm{CB}_{1} \mathrm{R}$ mRNA in the

renal cortex compared to wild-type littermates (Figure 2). Twelve weeks after STZ-treatment to 
induce hyperglycemia, body weight of the two strains remained similar, but wild-type mice had higher kidney weight (Figure 2A) and higher Cnr1 expression in renal cortices as compared to diabetic pCB1Rko mice (Figure 2B). Meanwhile, cortical levels of AEA and 2-AG remained similar in the two strains (Figure 2C).

Wild-type and pCB1Rko mice developed similar STZ-induced hyperglycemia and a similar increase in hAlc (Figure 2D). However, after 12 weeks of chronic hyperglycemia, pCB1Rko mice displayed significantly lower levels of blood urea nitrogen (BUN) and less albuminuria compared to their STZ-treated wild-type littermates (Figure 2E), suggesting better preserved glomerular and podocyte functions in the former. Correspondingly, pCB1Rko mice were protected from podocyte loss, as demonstrated by significant preservation of WT-1 and podocalyxin immunostaining (Figure 2F). In addition, diabetic wild-type mice displayed an increase in glomerular tuft area, which remained normal in diabetic pCB1Rko mice (Figure 2F). These histological observations were consistent with altered gene expression profiles. Indeed, the diabetes-induced decrease in the expression of genes encoding for structural podocyte proteins such as Nphs2, Nphs1 and zonula occludens (Tjp1) was absent in diabetic pCB1Rko mice (Figure 2G). Interestingly, the diabetes-induced increased expression of Des, which correlates with podocyte injury, was not influenced by the deletion of $\mathrm{CB}_{1} \mathrm{R}$ in podocytes (Figure 2G). Structural analysis by electron microscopy revealed mild segmental/variable thickening of glomerular basement membranes (GBM) with foot process fusion in the STZ-treated wild-type mice and focally shorter foot processes. In contrast, podocyte foot processes and GBMs were comparable between the citrate- and STZ-treated pCB1Rko mice although minor irregularities could be appreciated in the GBM thickness of STZ-treated pCB1Rko mice (Supplementary figure 2). 


\section{Podocyte-specific deletion of Cnr1 partially prevents proximal tubular cell injury and loss of}

\section{function.}

In addition to its protective effect on podocyte function, podocyte-specific deletion of Cnr1 also protected some tubular cell functions. For instance, pCB1Rko mice had lower urine output, less glycosuria and urinary $\mathrm{N}$-acetylglucosamine (NAG) activity, and increased expression of the proximal tubular cell marker megalin (Lrp2) in renal cortices compared to their wild-type littermates (Figure 3A). Interestingly, diabetic wild-type and pCB1Rko mice displayed similar increases in the expression of the sodium/glucose cotransporter 2 (SGLT2, Slc5a2) protein despite different levels of glycosuria (Figure 3A). Again, no differences in gene expression were observed under non-diabetic conditions except for a small increase in Lrp2 expression in pCB1Rko compared to wild-type mice.

Alterations in arginine metabolism, as reflected by increased expression of Arg2, results in vascular dysfunction in diabetes which contributes to the pathogenesis of DN [16]. We observed a strong increase in arginase- 2 protein in renal cortices from diabetic mice compared to nondiabetic wild-type mice, whereas no such increase was detectable in diabetic pCB1Rko mice (Figure 3B). Furthermore, lipocalin 2 has been linked to tubular cells apoptosis and renal lesion [17], and its protein and gene expression was strongly increased by diabetes in wild-type mice and much less so in pCB1Rko mice (Figure 3B). Renal cortical fibrosis was evident in diabetic wildtype but not pCB1Rko mice, as documented histologically by Picro-Sirius Red staining and by corresponding changes in the gene expression of pro-fibrotic markers Col1a, Timp1, Tgfb1 and Fn1 (Figure 3B). Furthermore, the commonly observed hyperglycemia-induced increase in caspase 3/7 activity was also attenuated in diabetic pCB1Rko compared to diabetic wild-type mice (Figure 3C). 


\section{Absence of $C_{1} R$ in podocytes prevents the diabetes-induced increase in oxidative stress and inflammation in renal cortices.}

In wild-type mice, diabetes was associated with increased ROS production in renal cortices, and corresponding increases in the expression of ROS-generating NADPH oxidase isoforms, such as NADPH oxidase4 (Nox4), p47phox and Nox1. On the other hand, no such diabetes-induced changes were detected in pCB1Rko mice (Figure 4A). Additionally, a strong infiltration of macrophages was evident in the glomeruli and interstitial space between proximal tubule cells of diabetic wild-type but not in pCB1Rko mice, as indicated by the ionized calcium binding adaptor molecule (Iba-1, microglia/macrophage-specific calcium binding protein) staining and by the parallel changes in the gene expression of macrophage markers Cd68 and F4/80 (Figure 4B). This macrophage infiltration, in turn, was associated with an increase in the gene expression of the proinflammatory markers Ccl2, Tnf, Il6 and Il18 but not Il1b (Figure 4C).

\section{Absence of $C_{B} R$ in podocytes mitigates diabetes-induced hypoxia and decrease in renal microcirculation.}

Microvascular density has been suggested as a component of CKD progression and as such may be impacted in diabetes $[18,19]$ and resulting in chronic hypoxia [20]. Because the genetic deletion of $\mathrm{CB}_{1} \mathrm{R}$ in podocytes had a protective effect on both glomerular and tubular functions, we explored the possibility of a parallel improvement in renal microcirculation, as assessed by Laser speckle contrast analysis (LASCA) [21, 22] and illustrated in Supplementary figure 3 where changes in blood flow are evident during renal ischemia/reperfusion. Under normal conditions, wild-type and pCB1Rko mice had similar levels of renal microcirculatory flux. However, diabetes in wild-type mice resulted in decreased renal microcirculation, while no such decrease was evident 
in pCB1Rko mice (Figure 4D). Additionally, in renal cortices from diabetic wild-type mice, the gene and protein expression of hypoxia-inducible factor- $\alpha$ (Hifla) was increased and that of vascular endothelial growth factor-A (Vegfa) reduced relative to control wild-type mice (Figure 4E). Furthermore, diabetic renal cortices displayed increased gene expression of the angiogenic factor thrombospondin-1 (Thsb1) and of the HIF-1 $\alpha$ target, NADH dehydrogenase-1 $\alpha$ subcomplex 4-like2 (Ndufa4l2) [23], as well as an increase in the lactate-to-pyruvate ratio, a marker of tissue hypoxia, relative to non-diabetic controls (Figure 4F). These diabetes-related changes were absent or attenuated in diabetic pCB1Rko mice, suggesting that podocyte $\mathrm{CB}_{1} \mathrm{R}$ activity plays a critical role in mediating the decrease in renal cortical blood flow, and thus, the resulting tissue hypoxia.

\section{Podocyte-derived factors regulate proximal tubular cell function}

To further explore the possible involvement of podocyte-derived factors in damaging tubular cell function, we prepared conditioned medium (CM) from podocytes exposed to high glucose for $48 \mathrm{~h}$. This $\mathrm{CM}$ was then supplemented with either vehicle (DMSO) or $\mathrm{CB}_{1} \mathrm{R}$ antagonist JD5037 and used to incubate renal proximal tubular cells (RPTC) for an additional $48 \mathrm{~h}$ in parallel with RPTC incubated in normal $(5 \mathrm{mM})$ or high glucose $(30 \mathrm{mM})$ media. Both high glucose and CM induced CNR1 expression in RPTC while the presence of JD5037 completely prevented this effect (Figure 5A). While high glucose alone could reduce megalin (LRP2) and increase lipocalin 2 (LCN2), vimentin (VIM-1) and arginase 2 (ARG2) expression suggesting RPTC impairment, podocyte-CM significantly exacerbated these effects, suggesting the involvement of podocyte-derived factors in promoting tubular dysfunction (Figure 5B). Remarkably, $\mathrm{CB}_{1} \mathrm{R}$ antagonism prevented the $\mathrm{CM}$ induced additional damage regarding $L R P 2, L C N 2$ and $A R G 2$ expression, while fully blocking the increase in VIM-1 (Figure 5B). Interestingly, the minimal effects of high glucose on RPTC's 
CCL2, TNF, IL18 and TGFB expression were significantly increased by exposure to podocyteCM, particularly for CCL2 expression, which was dramatically increased (Figure 5C). The potentiation of the effects of high glucose by podocyte-CM was fully or partially abrogated in the presence of JD5037 (Figure 5C).

\section{DISCUSSION}

Podocytes are essential components of the glomerular ultrafiltration apparatus, and their injury has been well established as a key pathogenic factor in the proteinuria and glomerulopathy associated with various forms of $\mathrm{CKD}$, including $\mathrm{DN}$. Recent evidence highlighted the role of endocannabinoids acting via $\mathrm{CB}_{1} \mathrm{R}$ in both type-1 $\mathrm{DN}$ [24] and type-2 $\mathrm{DN}$ [11, 25], although the specific role of $\mathrm{CB}_{1} \mathrm{R}$ in podocytes has remained unclear. Here we generated a podocyte-specific $\mathrm{CB}_{1} \mathrm{R}$ knockout mouse strain to determine whether activation of $\mathrm{CB}_{1} \mathrm{R}$ in podocytes significantly contributes to impaired glomerular functions, including albuminuria, in type-1 DN induced in mice by STZ treatment. Unexpectedly, we also found that activation of podocyte $\mathrm{CB}_{1} \mathrm{R}$ induces tubular dysfunction, fibrosis and the reduction of renal cortical microcirculation in STZ-induced DN. Lastly, we demonstrate that soluble factors released by podocytes in response to $\mathrm{CB}_{1} \mathrm{R}$ activation may mediate a crosstalk between podocytes and proximal tubular epithelial cells.

An upregulation of renal $\mathrm{CB}_{1} \mathrm{R}$ has been linked to the development of $\mathrm{DN}[10,11,24,25]$ and renal fibrosis [26]. In turn, $\mathrm{CB}_{1} \mathrm{R}$ antagonists have been identified as potential therapeutic tools, as the globally active $\mathrm{CB}_{1} \mathrm{R}$ antagonist rimonabant ameliorates albuminuria and reduces glomerular and tubular damage in rodent models of obesity/diabetes $[15,25]$ and also reduces albuminuria in STZ-treated mice with type-1 DN [24]. Although the clinical use of rimonabant is precluded by its CNS-mediated side effects, peripherally restricted $\mathrm{CB}_{1} \mathrm{R}$ antagonism is devoid of 
such undesired effects while still effective in maintaining and restoring podocyte integrity and functions in a rat model of type-2 $\mathrm{DN}[11]$. However, the cellular localization of $\mathrm{CB}_{1} \mathrm{R}$ responsible for these effects is not clear, as gluco- and/or lipotoxic stress increases $\mathrm{CB}_{1} \mathrm{R}$ expression not only in podocytes [15], but also in renal cortical mesangial cells [10] and proximal tubular cells [13, 27, 28], and $\mathrm{CB}_{1} \mathrm{R}$ activation leads to the apoptosis of both cell types [10,28].

In order to investigate the specific role of podocyte $\mathrm{CB}_{1} \mathrm{R}$ in $\mathrm{DN}$, we developed the first podocyte-specific $\mathrm{CB}_{1} \mathrm{R}$ knock-out mouse model. Podocyte-specific deletion of $\mathrm{CB}_{1} \mathrm{R}$ mitigated albuminuria and the increase in BUN in STZ-treated mice without affecting their hyperglycemia, indicating improved glomerular functions and better preserved podocyte integrity. Furthermore, STZ-induced tubular dysfunction, as indicated by polyuria, glycosuria, and decreased cortical expression of megalin, was also mitigated by the absence of $\mathrm{CB}_{1} \mathrm{R}$ in podocytes, suggesting that podocyte injury may influence tubular epithelial cell health and function in a $\mathrm{CB}_{1} \mathrm{R}$-dependent manner.

One possible explanation for this unexpected finding is that an improvement of glomerular filtration and reduction in albuminuria and other filtered tubulotoxins directly leads to decreased proximal tubulointerstitial damage [29-31]. We have found that the absence of $\mathrm{CB}_{1} \mathrm{R}$ from podocytes mitigated the hyperglycemia-induced lipocalin 2 overexpression in tubular cells, which may also be related to the decrease in albuminuria in diabetic pCB1Rko mice, as it was previously shown that an increase in albumin promotes tubular cell death by inducing $\mathrm{Ca}^{2+}$-dependent endoplasmic reticulum stress, leading to lipocalin 2 overexpression [17]. Similarly, an increase in albumin can induce TNF- $\alpha$, CCL2 and platelet-derived growth factor secretion by tubular cells, and these cytokines can serve as a trigger for the development of interstitial fibrosis $[32,33]$. This deleterious effect of albuminuria can be facilitated by the high glucose-mediated increase in 
metalloproteinase activity in tubular cells that leads to the shedding of the megalin/cubilin complex, which contributes to the impaired tubular clearance of filtered proteins including albumin $[34,35]$, as they are partially reabsorbed via megalin [36]. The tubular expression of megalin is reduced during obesity or diabetes and is restored by pharmacological inhibition of $\mathrm{CB}_{1} \mathrm{R}[11,37]$, as demonstrated in the present study.

Another explanation involves a paracrine interaction between podocytes and renal proximal tubular cells (RPTC). Injured podocytes are known to release various cytokines including TNF- $\alpha$, VEGFA, TGF- $\beta$ and IL-6 [38-40] which, in turn, may reach tubular cells and influence their activity. We have found that podocytes can release endocannabinoids when injured by exposure to high glucose or angiotensin II [11], and the released endocannabinoids could similarly act on neighboring cells, including RPTC. Indeed, RPTC express $\mathrm{CB}_{1} \mathrm{R}$ [37], which is upregulated in streptozotocin-induced type-1 diabetes [27] and its activation promotes RPTC hypertrophy [41], an early sign of DN. Furthermore, a recent report demonstrated a critical role for RPTC-expressed $\mathrm{CB}_{1} \mathrm{R}$ in the context of obesity-induced nephropathy [13] where it mediates RPTC inflammation and fibrosis. Here we demonstrated that incubating RPTC in conditioned medium from high glucose-exposed podocytes prompts an overexpression of the RPTC proteins involved in tubular damage, including TNF- $\alpha$, CCL2, vimentin-1, lipocalin 2 and arginase 2. In parallel, a decrease in megalin expression suggests tubular dysfunction. Moreover, these changes significantly exceeded those induced by high glucose alone, and were partially or completely abrogated in the presence of $100 \mathrm{nM}$ JD5037. This provides strong evidence for a previously unrecognized paracrine function of podocyte-derived endocannabinoids acting on $\mathrm{CB}_{1} \mathrm{R}$ of RPTC, inducing and aggravating tubular damage. However, we cannot exclude the possibility that factors released by 
podocytes might stimulate RPTC's to produce endocannabinoids, whose autocrine actions are then blocked by the $\mathrm{CB}_{1} \mathrm{R}$ antagonist.

In addition, a $\mathrm{CB}_{1} \mathrm{R}$-mediated increase in TGF- $\beta$ production by podocytes facilitates the synthesis of pro-fibrogenic proteins such as type IV collagen, fibronectin or metalloproteinase inhibitors [42]. Thus, podocyte- $\mathrm{CB}_{1} \mathrm{R}$ contributes to the induction of interstitial fibrosis and tubular atrophy [43]. Diabetic glomerular fibrosis involves the accumulation of extracellular matrix proteins in the mesangial interstitial space, resulting in either diffuse or nodular changes [44], and podocyte damage or loss leads to glomerulosclerosis [45]. $\mathrm{CB}_{1} \mathrm{R}$ exerts pro-fibrogenic action in various tissues [46, 47], including kidneys, where its genetic invalidation or pharmacological blockade markedly reduced renal fibrosis in mice with unilateral ureteral ligation [26], in which $\mathrm{CB}_{1} \mathrm{R}$ blockade reduced renal fibrosis by inhibiting macrophage infiltration by reducing CCL2 synthesis [26]. Similarly, pharmacological blockade of $\mathrm{CB}_{1} \mathrm{R}$ using rimonabant abolished the high glucose-mediated increase in profibrogenic genes in cultured podocytes [15]. These results agree with the profibrogenic role of podocyte $\mathrm{CB}_{1} \mathrm{R}$ and their additional involvement in the associated macrophage infiltration in both glomeruli and the interstitial space around tubular cells, which maintains a pro-inflammatory environment.

Contrary to our findings, Barutta et al reported that the overexpression of the pro-fibrotic proteins, TGF- $\beta$, fibronectin, and connective tissue growth factor in the cortex were unaltered by the $\mathrm{CB}_{1} \mathrm{R}$ antagonist rimonabant in STZ-treated mice [24]. However, in a more recent study using the same model, they found significant suppression of pro-fibrogenic gene and protein expression by the peripherally restricted neutral $\mathrm{CB}_{1} \mathrm{R}$ antagonist $\mathrm{AM} 6545$ [48]. Furthermore, in $d b / d b$ mice with type-2 $\mathrm{DN}, \mathrm{CB}_{1} \mathrm{R}$ blockade with rimonabant decreased albuminuria and suppressed the cortical expression of profibrotic (TGF- $\beta 1$, type IV collagen, PAI-1) and pro-inflammatory 
markers (CCL2) [15]. Together, these findings support the pro-fibrogenic role of $\mathrm{CB}_{1} \mathrm{R}$ in the kidney.

Primary glomerular disease disrupts blood flow into the interstitium due to changes in vascular tone. Such microvascular changes appear prior to the decline in kidney function in STZtreated rats [18]. Moreover, interstitial fibrosis and extracellular matrix accumulation impair oxygen diffusion to tubular and interstitial cells by increasing the distance between blood vessels and nearby cells [49], leading to tissue hypoxia. Our observations of diabetic wild-type kidney are compatible with advance stage renal cortical hypoxia, as evidenced by reduced cortical microcirculation, reduced Vegfa expression likely resulting from endothelial cell apoptosis [50], increased Hif1a, thbs1, ndufa4/2 gene expression and increased lactate-to-pyruvate ratio, which are all markers of tissue hypoxia. Strikingly, the absence of podocyte $\mathrm{CB}_{1} \mathrm{R}$ mitigated all of these diabetes-induced changes, suggesting a critical role of podocyte $\mathrm{CB}_{1} \mathrm{R}$ in the control of vascular endothelial function and ultimately cortical microcirculation. Such an effect may be mediated indirectly, through the pro-fibrogenic influence of podocyte $\mathrm{CB}_{1} \mathrm{R}$ that promotes tissue hypoxia. Additionally, activation of podocyte $\mathrm{CB}_{1} \mathrm{R}$ may result in the release of humoral factors that may have a paracrine damaging action on vascular endothelial cells. Although we have not tested such a cell-to-cell communication between podocytes and endothelial cells, podocytes- or endothelial cells-derived endocannabinoids may be involved, given our experimental results with conditioned media. The involvement of endocannabinoids is further suggested by published evidence that $\mathrm{CB}_{1} \mathrm{R}$ agonists suppress, while $\mathrm{CB}_{1} \mathrm{R}$ inhibitors increase, Vegf expression in a proinflammatory/fibrogenic environment $[51,52]$.

In conclusion, we have shown that podocyte $\mathrm{CB}_{1} \mathrm{R}$ play a key role in the impaired glomerular functions, proximal tubulopathy, interstitial fibrosis, and reduced microcirculation 
associated with DN. Importantly, tubular dysfunction may be mediated, in part, by the paracrine or autocrine action of endocannabinoids acting via $\mathrm{CB}_{1} \mathrm{R}$ on proximal tubular cells as schematically illustrated in Figure 5D.

\section{METHODS}

For a detailed description of materials and methods used, see supplementary methods.

\section{Animals and induction of diabetes}

All animal protocols conformed to the National Institutes of Health (NIH) guidelines and were approved by the Institutional Animal Care and Use Committee of the National Institute on Alcohol Abuse and Alcoholism.

\section{Renal microcirculation}

Renal microcirculation was assessed by Laser speckle contrast analysis (LASCA) using the moorFLPI-2 blood flow imager (Moor Instruments, Wilmington, DE, USA).

\section{Statistics}

Values are expressed as means \pm SEM. Data were analyzed by ANOVA followed by the Tukey-

Kramer post-hoc test. Time-dependent variables were analyzed and results in multiple groups were compared by ANOVA followed by the Bonferroni test. (GraphPad Prism version 7 for Windows, San Diego, CA). Significance was set at $P<0.05$.

\section{AUTHOR CONTRIBUTIONS}

T.J. and G.K. designed the study, analyzed results and wrote the manuscript; T.J. performed most of the experiments; J.K.P. and G.G. assisted with animal care and in vivo experiments; Z.V.V and J.P. ran the microcirculation experiments; N.J.C. assisted with cell culture and PCR analyses; A.Z.R. performed the renal pathology analyses; R.C. ran the endocannabinoid measurements; K.M. kindly provided the L15 CB1R antibody; P.P. provided insights and critical review of the manuscript. All authors had access to the manuscript and agreed with the final version. 


\section{ACKNOWLEDGMENTS}

All authors declare no conflict of interest. We thank Patricia M. Zerfas (Office of Research Services, NIH) for technical help with electron microscopy; Judith Harvey-White (NIAAA, NIH) for technical assistance; Luo Guoxiang (NIAAA, NIH) for assistance with animal breeding and genotyping; Raouf Kechrid (NIAAA, NIH) for assistance with the animal studies; and Dr. Joshua Rein (Mount Sinai Hospital) for helpful and constructive comments.

This study was supported by intramural NIH funds to G.K. 


\section{REFERENCES}

[1] Reidy K, Kang HM, Hostetter T, Susztak K. Molecular mechanisms of diabetic kidney disease. J Clin Invest. 2014; 124: 2333-2340

[2] Cao Z, Cooper ME. Pathogenesis of diabetic nephropathy. Journal of Diabetes Investigation. 2011; 2: 243-247

[3] Pavenstädt H, Kriz W, Kretzler M. Cell Biology of the Glomerular Podocyte. Physiological Reviews. 2003; 83: 253

[4] Assady S, Wanner N, Skorecki KL, Huber TB. New Insights into Podocyte Biology in Glomerular Health and Disease. Journal of the American Society of Nephrology : JASN. 2017; 28: $1707-1715$

[5] Fu J, Lee K, Chuang PY, Liu Z, He JC. Glomerular endothelial cell injury and cross talk in diabetic kidney disease. American journal of physiology Renal physiology. 2015; 308: F287297

[6] Wiggins RC. The spectrum of podocytopathies: A unifying view of glomerular diseases. Kidney international. 2007; 71: 1205-1214

[7] Mazier W, Saucisse N, Gatta-Cherifi B, Cota D. The Endocannabinoid System: Pivotal Orchestrator of Obesity and Metabolic Disease. Trends in Endocrinology \& Metabolism. 2015; 26: $524-537$

[8] Hryciw DH, McAinch AJ. Cannabinoid receptors in the kidney. Curr Opin Nephrol Hypertens. 2016; 25: 459-464

[9] Tam J. The emerging role of the endocannabinoid system in the pathogenesis and treatment of kidney diseases. Journal of basic and clinical physiology and pharmacology. 2016; 27: 267-276 [10] Lim JC, Lim SK, Park MJ, Kim GY, Han HJ, Park SH. Cannabinoid receptor 1 mediates high glucose-induced apoptosis via endoplasmic reticulum stress in primary cultured rat mesangial cells. American Journal of Physiology - Renal Physiology. 2011; 301: F179

[11] Jourdan T, Szanda G, Rosenberg AZ, et al. Overactive cannabinoid 1 receptor in podocytes drives type 2 diabetic nephropathy. Proceedings of the National Academy of Sciences. 2014; 111: E5420-E5428

[12] Sampaio LS, Taveira Da Silva R, Lima D, et al. The endocannabinoid system in renal cells: regulation of $\mathrm{Na}(+)$ transport by $\mathrm{CB} 1$ receptors through distinct cell signalling pathways. British journal of pharmacology. 2015; 172: 4615-4625

[13] Udi S, Hinden L, Earley B, et al. Proximal Tubular Cannabinoid-1 Receptor Regulates Obesity-Induced CKD. Journal of the American Society of Nephrology : JASN. 2017; DOI: 10.1681/asn.2016101085

[14] Deutsch DG, Goligorsky MS, Schmid PC, et al. Production and physiological actions of anandamide in the vasculature of the rat kidney. J Clin Invest. 1997; 100: 1538-1546

[15] Nam DH, Lee MH, Kim JE, et al. Blockade of cannabinoid receptor 1 improves insulin resistance, lipid metabolism, and diabetic nephropathy in $\mathrm{db} / \mathrm{db}$ mice. Endocrinology. 2012; 153: 1387-1396

[16] Morris SM, Gao T, Cooper TK, Kepka-Lenhart D, Awad AS. Arginase-2 Mediates Diabetic Renal Injury. Diabetes. 2011; 60: 3015-3022

[17] El Karoui K, Viau A, Dellis O, et al. Endoplasmic reticulum stress drives proteinuriainduced kidney lesions via Lipocalin 2. Nature communications. 2016; 7: 10330 
[18] Maric-Bilkan C, Flynn ER, Chade AR. Microvascular disease precedes the decline in renal function in the streptozotocin-induced diabetic rat. American Journal of Physiology - Renal Physiology. 2012; 302: F308

[19] Nakagawa T, Kosugi T, Haneda M, Rivard CJ, Long DA. Abnormal angiogenesis in diabetic nephropathy. Diabetes. 2009; 58: 1471-1478

[20] Long DA, Norman JT, Fine LG. Restoring the renal microvasculature to treat chronic kidney disease. Nat Rev Nephrol. 2012; 8: 244-250

[21] Bezemer R, Legrand M, Klijn E, et al. Real-time assessment of renal cortical microvascular perfusion heterogeneities using near-infrared laser speckle imaging. Opt Express. 2010; 18: 1505415061

[22] Holstein-Rathlou NH, Sosnovtseva OV, Pavlov AN, Cupples WA, Sorensen CM, Marsh DJ. Nephron blood flow dynamics measured by laser speckle contrast imaging. American journal of physiology Renal physiology. 2011; 300: F319-329

[23] Tello D, Balsa E, Acosta-Iborra B, et al. Induction of the mitochondrial NDUFA4L2 protein by HIF-1alpha decreases oxygen consumption by inhibiting Complex I activity. Cell metabolism. 2011; 14: 768-779

[24] Barutta F, Corbelli A, Mastrocola R, et al. Cannabinoid receptor 1 blockade ameliorates albuminuria in experimental diabetic nephropathy. Diabetes. 2010; 59: 1046-1054

[25] Janiak P, Poirier B, Bidouard JP, et al. Blockade of cannabinoid CB1 receptors improves renal function, metabolic profile, and increased survival of obese Zucker rats. Kidney Int. 2007; 72: $1345-1357$

[26] Lecru L, Desterke C, Grassin-Delyle S, et al. Cannabinoid receptor 1 is a major mediator of renal fibrosis. Kidney Int. 2015; 88: 72-84

[27] Jenkin KA, McAinch AJ, Zhang Y, Kelly DJ, Hryciw DH. Elevated cannabinoid receptor 1 and $\mathrm{G}$ protein-coupled receptor 55 expression in proximal tubule cells and whole kidney exposed to diabetic conditions. Clin Exp Pharmacol Physiol. 2015; 42: 256-262

[28] Lim JC, Lim SK, Han HJ, Park SH. Cannabinoid receptor 1 mediates palmitic acid-induced apoptosis via endoplasmic reticulum stress in human renal proximal tubular cells. J Cell Physiol. 2010; 225: 654-663

[29] Schlondorff DO. Overview of factors contributing to the pathophysiology of progressive renal disease. Kidney Int. 2008; 74: 860-866

[30] Mundel P, Reiser J. Proteinuria: an enzymatic disease of the podocyte? Kidney international. 2010; 77: 571-580

[31] Menzel S, Moeller MJ. Role of the podocyte in proteinuria. Pediatric Nephrology (Berlin, Germany). 2011; 26: 1775-1780

[32] Rangan GK, Wang Y, Tay Y-C, Harris DCH. Differential effects of albumin on cytokine gene expression in proximal tubular epithelial cells. Nephrology Dialysis Transplantation. 2005; 20: $1013-1014$

[33] Tang S, Leung JCK, Abe K, et al. Albumin stimulates interleukin-8 expression in proximal tubular epithelial cells in vitro and in vivo. Journal of Clinical Investigation. 2003; 111: 515-527

[34] Liu D, Wen Y, Tang T-T, et al. Megalin/Cubulin-Lysosome-mediated Albumin Reabsorption Is Involved in the Tubular Cell Activation of NLRP3 Inflammasome and Tubulointerstitial Inflammation. Journal of Biological Chemistry. 2015; 290: 18018-18028

[35] Biemesderfer D. Regulated intramembrane proteolysis of megalin: linking urinary protein and gene regulation in proximal tubule? Kidney Int. 2006; 69: 1717-1721 
[36] Cui S, Verroust PJ, Moestrup SK, Christensen EI. Megalin/gp330 mediates uptake of albumin in renal proximal tubule. American Journal of Physiology - Renal Fluid and Electrolyte Physiology. 1996; 271: F900-F907

[37] Tam J, Cinar R, Liu J, et al. Peripheral cannabinoid-1 receptor inverse agonism reduces obesity by reversing leptin resistance. Cell metabolism. 2012; 16: 167-179

[38] Shankland SJ. The podocyte's response to injury: role in proteinuria and glomerulosclerosis. Kidney Int. 2006; 69: 2131-2147

[39] Lee HS, Song CY. Effects of TGF- $\beta$ on Podocyte Growth and Disease Progression in Proliferative Podocytopathies. Kidney and Blood Pressure Research. 2010; 33: 24-29

[40] Bartlett CS, Jeansson M, Quaggin SE. Vascular Growth Factors and Glomerular Disease. Annual review of physiology. 2016; 78: 437-461

[41] Jenkin KA, McAinch AJ, Grinfeld E, Hryciw DH. Role for cannabinoid receptors in human proximal tubular hypertrophy. Cell Physiol Biochem. 2010; 26: 879-886

[42] Li JH, Huang XR, Zhu HJ, Johnson R, Lan HY. Role of TGF-beta signaling in extracellular matrix production under high glucose conditions. Kidney Int. 2003; 63: 2010-2019

[43] Burns WC, Velkoska E, Dean R, Burrell LM, Thomas MC. Angiotensin II mediates epithelial-to-mesenchymal transformation in tubular cells by ANG 1-7/MAS-1-dependent pathways. American Journal of Physiology - Renal Physiology. 2010; 299: F585

[44] Alsaad KO, Herzenberg AM. Distinguishing diabetic nephropathy from other causes of glomerulosclerosis: an update. Journal of clinical pathology. 2007; 60: 18-26

[45] Wharram BL, Goyal M, Wiggins JE, et al. Podocyte depletion causes glomerulosclerosis: diphtheria toxin-induced podocyte depletion in rats expressing human diphtheria toxin receptor transgene. Journal of the American Society of Nephrology : JASN. 2005; 16: 2941-2952

[46] Cinar R, Iyer MR, Liu Z, et al. Hybrid inhibitor of peripheral cannabinoid-1 receptors and inducible nitric oxide synthase mitigates liver fibrosis. JCI Insight. 2016; 1: e87336

[47] Cinar R, Gochuico BR, Iyer MR, et al. Cannabinoid CB1 receptor overactivity contributes to the pathogenesis of idiopathic pulmonary fibrosis. JCI Insight. 2017; 2: pii: 92281

[48] Barutta F, Grimaldi S, Gambino R, et al. Dual therapy targeting the endocannabinoid system prevents experimental diabetic nephropathy. Nephrology, dialysis, transplantation : official publication of the European Dialysis and Transplant Association - European Renal Association. 2017; 32: 1655-1665

[49] Ohashi R, Kitamura H, Yamanaka N. Peritubular capillary injury during the progression of experimental glomerulonephritis in rats. Journal of the American Society of Nephrology. 2000; 11: $47-56$

[50] Kang DH, Joly AH, Oh SW, et al. Impaired angiogenesis in the remnant kidney model: I. Potential role of vascular endothelial growth factor and thrombospondin-1. Journal of the American Society of Nephrology. 2001; 12: 1434-1447

[51] Staiano RI, Loffredo S, Borriello F, et al. Human lung-resident macrophages express CB1 and $\mathrm{CB} 2$ receptors whose activation inhibits the release of angiogenic and lymphangiogenic factors. J Leukoc Biol. 2016; 99: 531-540

[52] Sathyapalan T, Javed Z, Kilpatrick ES, Coady AM, Atkin SL. Endocannabinoid receptor blockade increases vascular endothelial growth factor and inflammatory markers in obese women with polycystic ovary syndrome. Clinical endocrinology. 2017; 86: 384-387 


\section{LEGENDS}

Figure 1: High-glucose mediated effects on endocannabinoid tone, podocyte health and inflammatory status of human podocytes.

A) CNR1 and CNR2 gene expression, anandamide (AEA), 2-arachidonoyl glycerol (2-AG) and arachidonic acid in podocytes exposed to either 5 (white bars) or $30 \mathrm{mM}$ (black bars) glucose for 24-48-72h. B) Podocin (NPHS2), nephrin (NPHS1) and desmin (DES) gene expression in podocytes exposed to either 5 (white bars) or $30 \mathrm{mM}$ (black bars) glucose for 24-48-72h. C-D) Chemokine ligand 2 (CCL2), tumor necrosis factor- $\alpha$ (TNF- $\alpha$ ), interleukin-18 (IL-18) protein secretion and transforming growth factor $\beta 1$ (TGFB1) gene expression in podocytes exposed to either 5 (white bars) or $30 \mathrm{mM}$ (black bars) glucose for 24-48-72h. E) CNR1 and CNR2 gene expression in human podocytes exposed to 5 (white bars) or $30 \mathrm{mM}$ glucose for $48 \mathrm{~h}$ in presence of control-siRNA (Ctrl, black bars) or CNR1-siRNA (grey bars). F) Podocin (NPHS2), nephrin (NPHS1) and desmin (DES) gene expression in podocytes exposed to 5 (white bars) or $30 \mathrm{mM}$ glucose for $48 \mathrm{~h}$ in presence of control-siRNA (Ctrl, black bars) or CNRl-siRNA (grey bars). G) Chemokine ligand 2 (CCL2), tumor necrosis factor $\alpha$ (TNF- $\alpha$ ), interleukin-18 (IL-18) protein secretion and transforming growth factor $\beta 1$ (TGFB1) gene expression in podocytes exposed to 5 (white bars) or $30 \mathrm{mM}$ glucose for $48 \mathrm{~h}$ in presence of control-siRNA (Ctrl, black bars) or CNR1siRNA (grey bars). Columns and bars represent means \pm SEM from 2 independent experiments with 6 replicates each. Significant differences from values in $5 \mathrm{mM}$ glucose $* \mathrm{P}<0.05, * * \mathrm{P}<0.01$, $* * * \mathrm{P}<0.001$ or $\mathrm{Ctrl}-\mathrm{siRNA}$ treated cells $\# \mathrm{P}<0.05$, \#\#P<0.01, \#\#\# $\mathrm{P}<0.001$. 

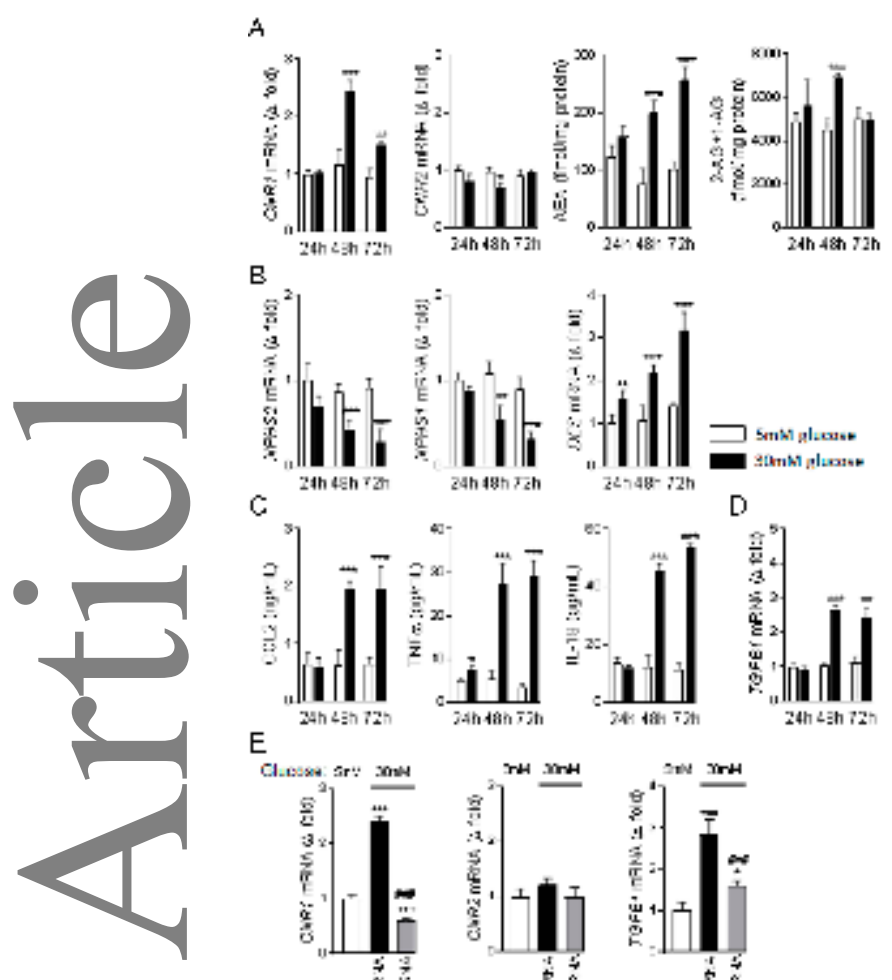

C

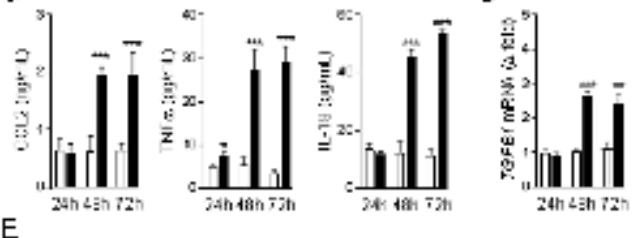

E
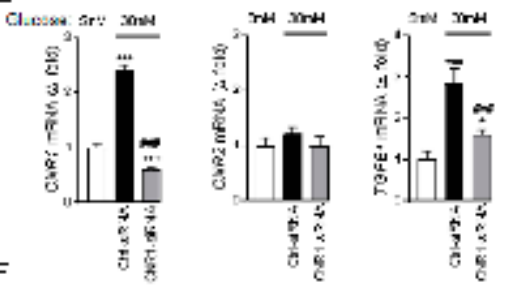

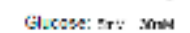

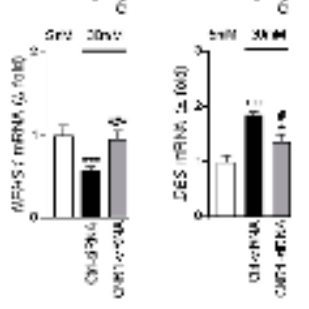

G

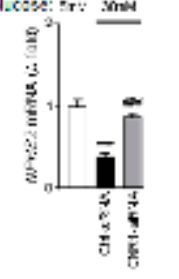

in

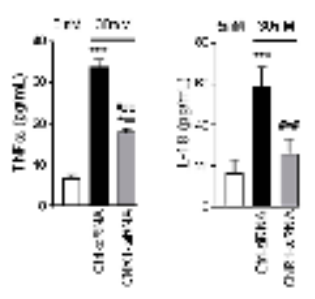


Figure 2: Effect of the streptozotocin-mediated hyperglycemia on ECS activity and glomerular function of pCB1Rko mice.

A) Body and kidney weight along with kidney/body weight ratio. B) Cnr1 gene expression in renal cortices. C) Anandamide (AEA), 2-arachidonoyl glycerol (2-AG) content of renal cortices. D) Blood glucose and glycated hemoglobin in citrate buffer-treated wild-type (open blue circles or blue columns, $\mathrm{n}=10$ or 4 respectively) or pCB1Rko mice (open red circles or red columns, $\mathrm{n}=10$ or 3 respectively), and streptozotocin-treated wild-type (open blue triangles or blue hatched columns, $\mathrm{n}=12$ or 5 respectively) or pCB1Rko mice (open red triangles or red hatched columns, $\mathrm{n}=15$ or 3 respectively). E) BUN and albuminuria in citrate buffer-treated wild-type (blue columns, $\mathrm{n}=10$ ) or pCB1Rko mice (red columns, $\mathrm{n}=10$ ), and streptozotocin-treated wild-type (blue hatched columns, $n=12$ ) or pCB1Rko mice (red hatched columns, n=15). F) Podocyte numbers assessed by immunohistochemistry using Wilms' Tumor 1 (WT-1) or podocalyxin staining to evaluate the number of nuclei and podocyte membrane integrity (scale bars $50 \mu \mathrm{m}$ ). Glomerular tuft area quantification after PAS staining. G) Gene expression for podocin (Nphs2), nephrin (Nphs1), Zonula occludens (Tjp1) and desmin (Des) in citrate buffer-treated wild-type (blue columns, $\mathrm{n}=10$ ) or pCB1Rko mice (red columns, $\mathrm{n}=5$ ), and streptozotocin-treated wild-type (blue hatched columns, $n=12$ ) or pCB1Rko mice (red hatched columns, $n=15$ ). Columns and bars represent means \pm SEM. Significant differences from values in citrate buffer-treated wild-type mice ${ }^{*} \mathrm{P}<0.05,{ }^{*} \mathrm{P}<0.01,{ }^{* * *} \mathrm{P}<0.001$ or streptozotocin-treated wild-type mice $\# \mathrm{P}<0.05, \# \# \mathrm{P}<0.01$, \#\#\# $\mathrm{P}<0.001$. 


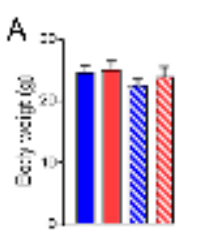

D
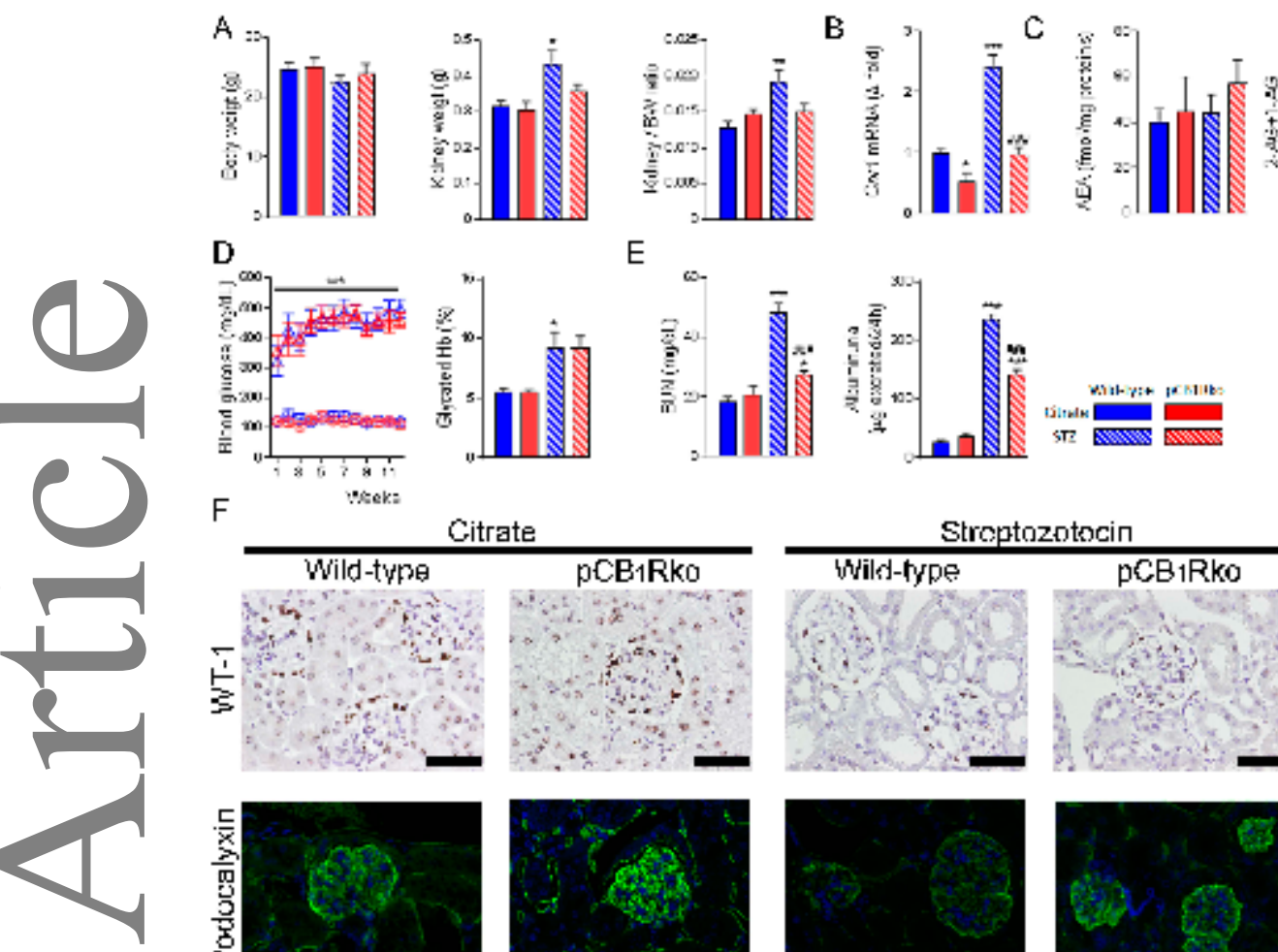

$\mathrm{E}$

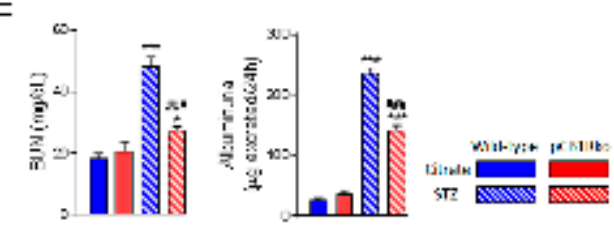

Citrate
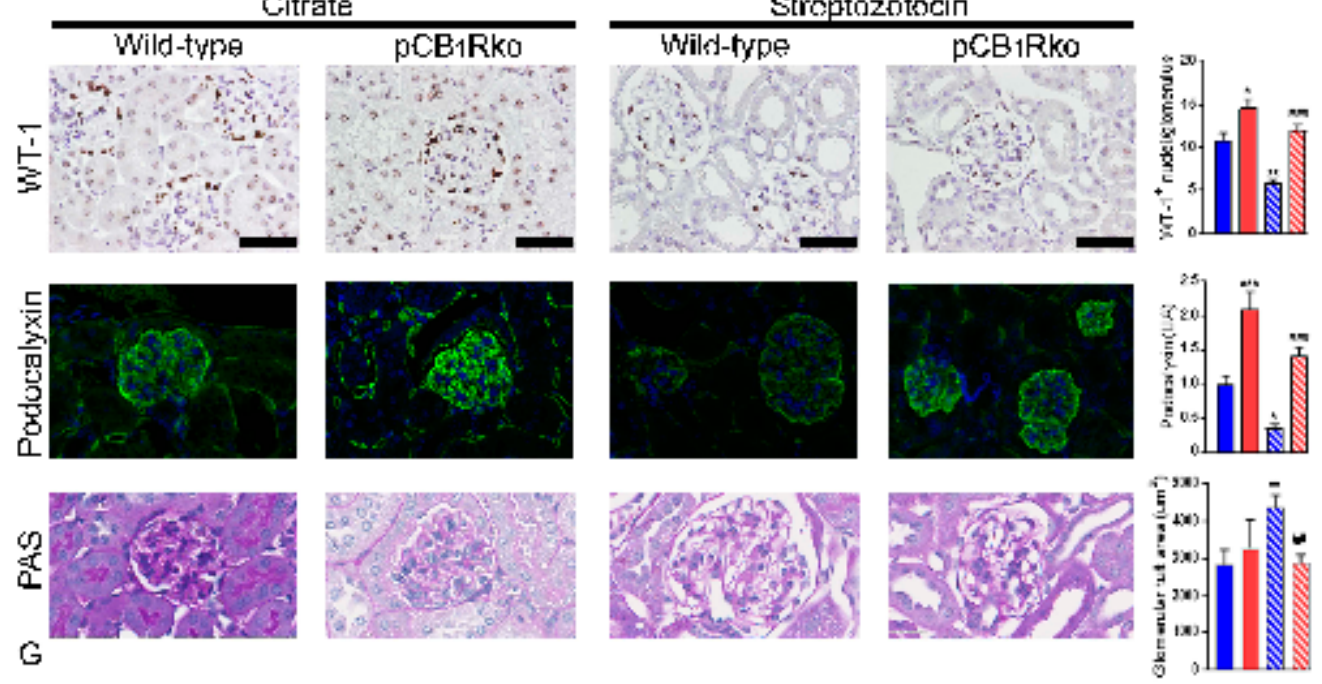
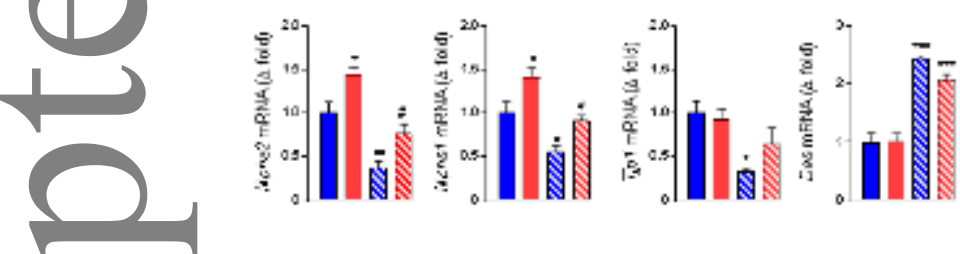

Figure 3: Effects of Cnr1 deletion in podocytes on proximal tubular cell injury and loss of function.

A) Urine output, glycosuria, urinary $\mathrm{N}$-acetyl- $\beta$-D-glucosaminidase (NAG) activity, cortical gene expression of megalin (Lrp2) and sodium-glucose co-transporter 2 (SGLT2 Slc5a2). B) Arginase2 and lipocalin-2 protein expression analysis by immuno-staining (scale bars $100 \mu \mathrm{m}$ ), and fibrosis assessment by Picro-Sirius red staining (scale bars $100 \mu \mathrm{m}$ ) and cortical gene expression of 
collagen 1 (Col1a), metallopeptidase inhibitor 1 (Timp1), TGF- $\beta$ (Tgfb1) and fibronectin (Fn-1). C) Caspase 3/7 activity in renal cortices from citrate buffer-treated wild-type (blue columns, $n=10$ ) or pCB1Rko mice (red columns, $\mathrm{n}=10$ ), and streptozotocin-treated wild-type (blue hatched columns, $n=12$ ) or pCB1Rko mice (red hatched columns, $n=15$ ). Columns and bars represent means \pm SEM. Significant differences from values in citrate buffer-treated wild-type mice ${ }^{*} \mathrm{P}<0.05,{ }^{*} \mathrm{P}<0.01, * * * \mathrm{P}<0.001$ or streptozotocin-treated wild-type mice $\# \mathrm{P}<0.05$, \#\#P<0.01, \#\# $\mathrm{P}<0.001$.

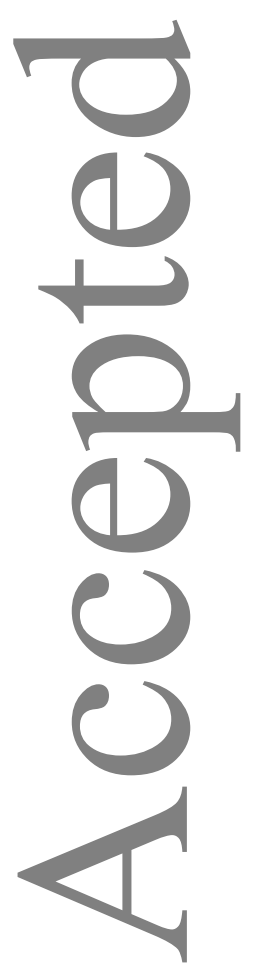




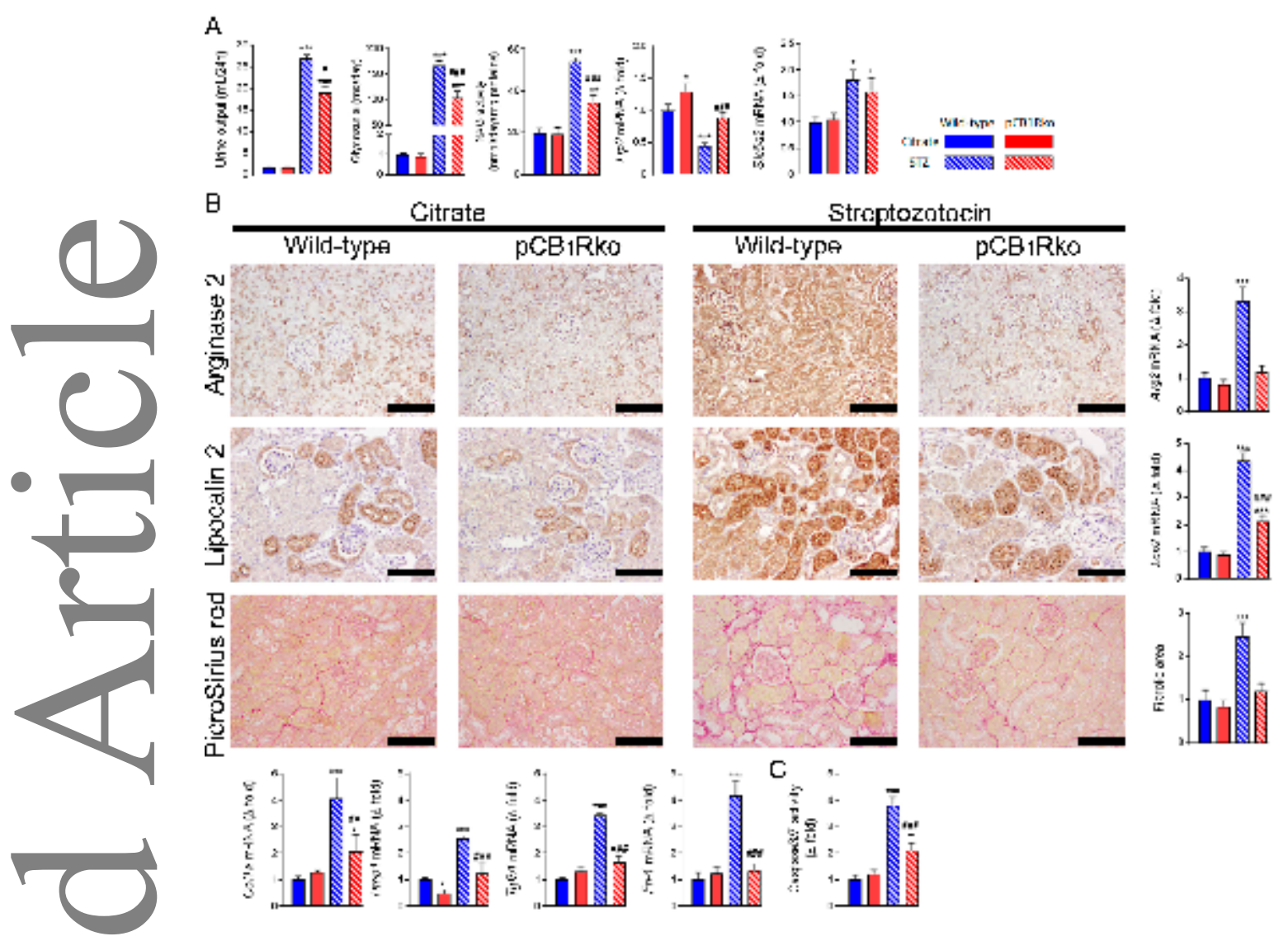

Figure 4: Cnr1 deletion in podocytes reduces oxidative stress, macrophages infiltration and renal inflammation while improving renal microcirculation.

A) Reactive oxygen species (ROS) quantification in renal cortices and gene expression of NADPH oxidase 4 (Nox4), NADPH oxidase 1 (Nox1) and neutrophil cytosolic factor 1 (p47phox). B) Macrophage infiltration as assessed by Iba-1 immuno-staining (scale bars: $100 \mu \mathrm{m}$ ) and cortical gene expression of the macrophages markers CD68 (Cd68) F4/80. C) Gene expression of CCL2 
(Ccl2), TNF- $\alpha$ (Tnf), IL-6 (Il6), IL-1 $\beta$ (Il1b) and IL-18 (Il18) in renal cortices from citrate buffertreated wild-type (blue columns, $\mathrm{n}=10$ ) or pCB1Rko mice (red columns, $\mathrm{n}=10$ ), and streptozotocin-treated wild-type (blue hatched columns, $\mathrm{n}=12$ ) or pCB1Rko mice (red hatched columns, $\mathrm{n}=15$ ). D) Representative illustration of renal microcirculation assessment by Laser speckle contrast analysis. E) Immunoreactive HIF- $1 \alpha$ and VEGF-A protein expression and mRNA levels in renal cortical sections; thrombospondin-1 (Thbs1) and NADH dehydrogenase [ubiquinone] 1 alpha subcomplex, 4-like 2 (Ndufa4l2) gene expression is also shown. F) Pyruvate, lactate concentration and lactate/pyruvate ratio in renal cortices from citrate buffer-treated wildtype (blue columns, $n=10$ ) or pCB1Rko mice (red columns, $n=10$ ), and streptozotocin-treated wild-type (dark hatched columns, $n=12$ ) or pCB1Rko mice (red hatched columns, $n=15$ ). Columns and bars represent means \pm SEM. Significant differences from values in citrate buffer-treated wildtype mice $* \mathrm{P}<0.05,{ }^{* *} \mathrm{P}<0.01,{ }^{* * *} \mathrm{P}<0.001$ or streptozotocin-treated wild-type mice $\# \mathrm{P}<0.05$, $\# \# \mathrm{P}<0.01$, \#\# $\mathrm{P}<0.001$. 

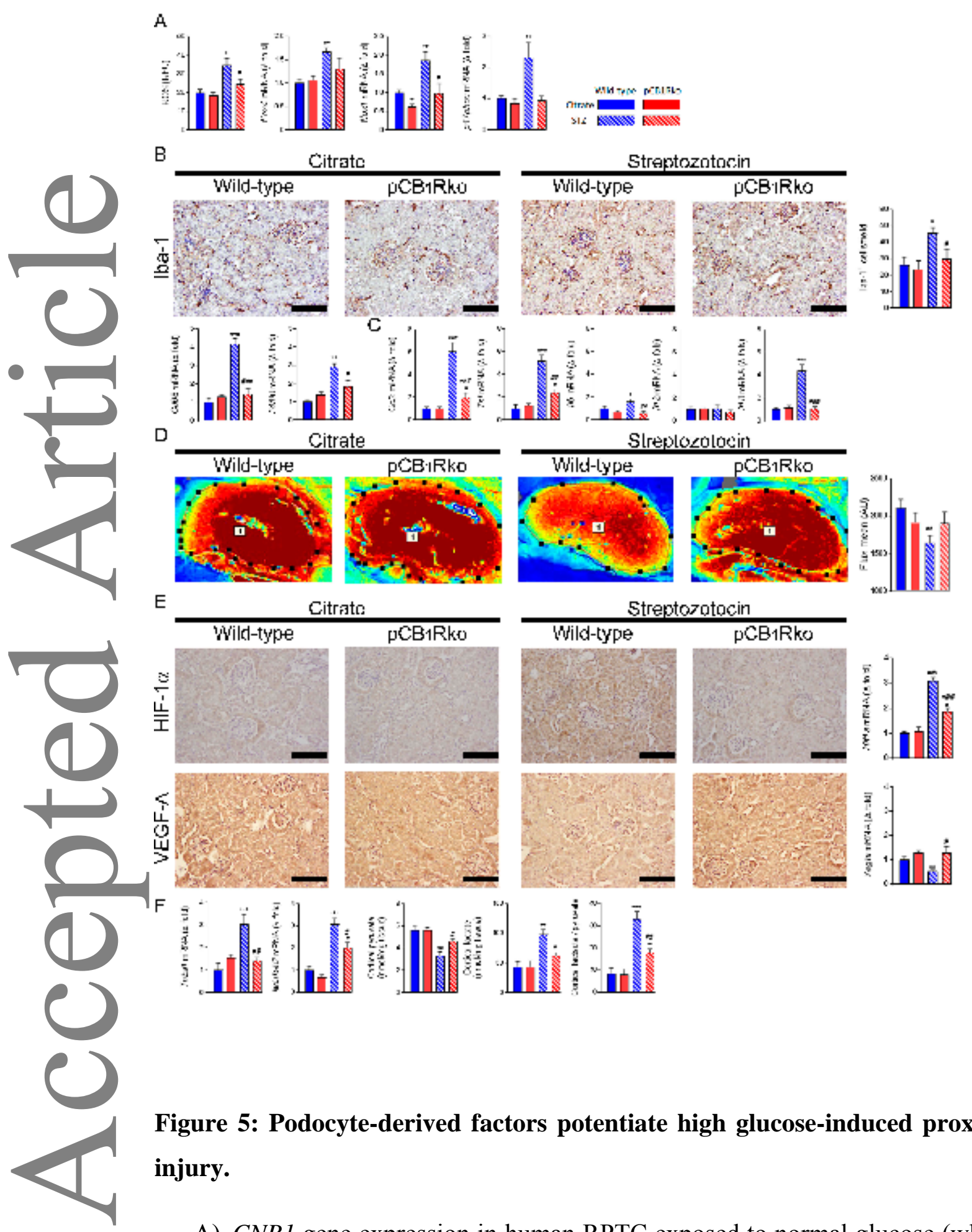

Figure 5: Podocyte-derived factors potentiate high glucose-induced proximal tubular cell injury.

A) CNR1 gene expression in human RPTC exposed to normal glucose (white), high glucose (black), podocyte-conditioned medium (green) or podocyte-conditioned medium containing $100 \mathrm{nM}$ JD5037 (green with white hatch). B) LRP2, LCN2, VIM-1 and ARG2 gene expression in RPTC exposed to normal glucose (white), high glucose (black), 
podocyte-conditioned medium (green) or podocyte-conditioned medium with JD5037 (green with white hatch). C) CCL2, TNF, IL18 and TGFB gene expression in RPTC exposed to normal glucose (white), high glucose (black), podocyte-conditioned medium (green) or podocyte-conditioned medium with JD5037 (green with white hatch). D) Schematic illustration of the proposed mechanism by CB1R-expressed podocytes mitigate podocyte and tubular injury. This illustration was prepared using a template on the Servier medical art website (http://www.servier.com/Powerpoint-image-bank). Columns and bars represent means \pm SEM from 2 independent experiments and 6 replicates per group. Significant differences from values in RPTC in normal glucose, ${ }^{*} \mathrm{P}<0.05,{ }^{* *} \mathrm{P}<0.01, * * *$ $\mathrm{P}<0.001$. Significance of the effect of JD5037 in RPTC in high glucose, $\# \mathrm{P}<0.05$, $\# \# \mathrm{P}<0.01$, \#\#\# $\mathrm{P}<0.001$. Significance of the effect of conditioned medium in RPTC in high glucose $\& \mathrm{P}<0.05, \& \& \mathrm{P}<0.01, \& \& \& \mathrm{P}<0.001$. 


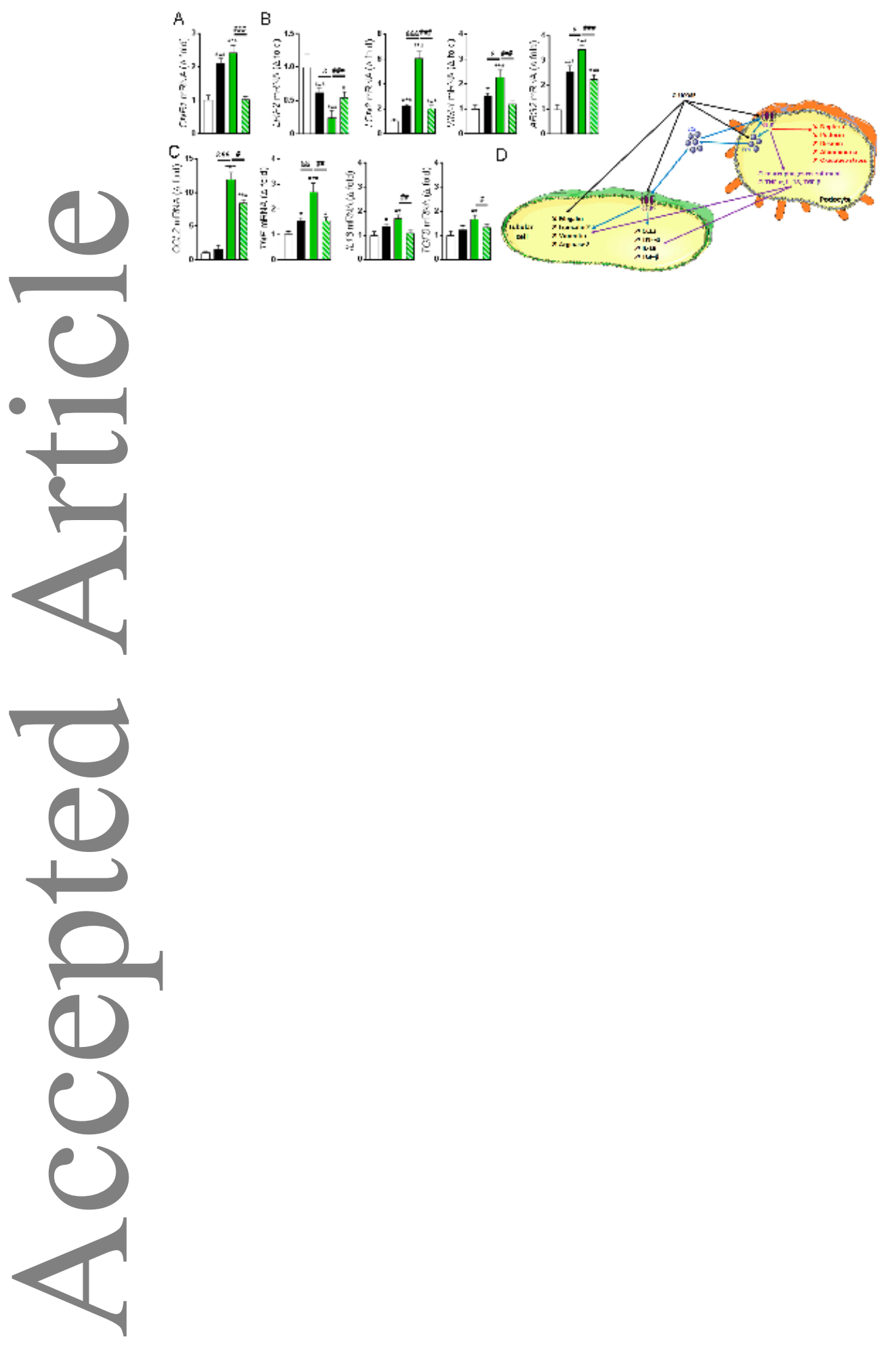

This article is protected by copyright. All rights reserved. 